\title{
MECHANICAL CHARACTERIZATION OF ORTHOTROPIC ELASTIC PARAMETERS OF A FOAM BY THE MIXED EXPERIMENTAL-NUMERICAL ANALYSIS
}

\author{
Monika Chuda-Kowalska, Tomasz Gajewski, Tomasz Garbowski \\ Poznan University of Technology, Institute of Structural Engineering, Poznań, Poland \\ e-mail: monika.chuda-kowalska@put.poznan.pl; tomasz.gajewski@put.poznan.pl; tomasz.garbowski@put.poznan.pl
}

\begin{abstract}
Different kind of foams, usually made from polymers, metals, ceramics, glass, etc. have been widely used in various branches of civil engineering since the $80 \mathrm{~s}$. The most common are polyurethane foams. Since their role in construction nowadays is not only to act as a thermal barrier but also to take some of loads, the engineers need to know also their mechanical properties. This implies that manufacturers or designers must perform a number of laboratory tests in order to find a set of substantial parameters of this particular material. Due to noticeable orthotropic behavior of foams, one needs to carry out several laboratory tests to identify elastic properties only. Here, an enhanced testing methodology is proposed to reduce the number of tests required for characterization of elastic orthotropic properties of foams. By combining the advanced measurement techniques, non-traditional experimental setup, numerical modeling and inverse analysis one can capture all nine elastic properties from just two or three tests. In the paper, full experimental and numerical procedures are presented and validated by noisy pseudo-experimental data.
\end{abstract}

Keywords: orthotropic elastic foams, digital image correlation, inverse analysis

\section{Introduction}

A cellular structure is made up of an interconnected network of solid struts and walls which form edges and faces of cells. This kind of materials appears widely in nature, and these days are also manufactured on a large scale. To produce cellular materials from metals, ceramics, polymers or composites, new technologies have been developed and successfully employed by many industries. For example, in the work by Gibson and Ashby (1997) a comprehensive description of structural, mechanical and thermal properties of a cellular material and its constituents is presented.

The simplest structure of a cellular material is a two-dimensional cellular solid called honeycomb often found as a sandwich shell in aircraft and offshore engineering. In the civil engineering, however, more common are three-dimensional cellular materials, whose cells are polyhedrons. In general, the 3D structure of a foam can have open or closed-cells. The first one has a solid material only in the cell edges. The second one has the solid material in the edges and faces of cells. All closed cells may contain gas which is produced through physical and biochemical processes during production.

For engineering purposes, cellular solids as foams contain an attractive set of features compared to solids. One of the most important is low density leading to the most common application, which is thermal insulation. A large capacity of compressive strains make the foams very attractive as energy-absorption structures (Ozturk and Anlas, 2009). Low densities of the foam used in sandwich panels allows one to design light and relatively stiff structures. In this case, the design process is connected to optimization because the structural modeling of sandwich panels 
is a complex task and depends on various factors (Błaszczuk and Pozorski, 2012; Studziński et al., 2013; Valdevit et al., 2004).

Using porous materials in structural applications (e.g. as a core in three-layered panels), knowledge about their mechanical behavior on both micro- and a macroscopic scale is required (Mills, 2007). Cellular materials models can be roughly divided into two categories: (1) micromechanical models and (2) phenomenological models. The first group of models is based on the analysis of mechanisms of deformation of the micro-cell structure under certain loading conditions. The most known micro-mechanical models for an open and a closed-cell foam with cubic symmetry are described by Gibson and Ashby (1997), Kordzikowski et al. (2005), JanusMichalska (2009), Murin et al. (2011). The micro-mechanical methods used to determine stiffness and strength properties of foams with tetradecahedral unit cells were evaluated by Subramanian and Sankar (2012). An approach to assess the macro-mechanical properties of open-cell foams using the micro-mechanical modeling can be found in the work of Janus-Michalska and Pęcherski (2003). On the other hand, the main goal of the phenomenological models is to fit the best to experimental mechanical behavior without direct relationship to physics of the phenomenon. Such models were commonly used and evaluated by many authors (Liu and Subhash, 2004; Avalle et al., 2007).

Ozturk and Anlas (2009) observed that the stress-strain relationship in the elastic range as well as unloading slope change with the number of loading cycles (density-dependent behavior). However, the stress-strain curve in the inelastic region remains unchanged. Therefore, they proposed a constitutive model capable to capture all these phenomena. Goga in (Murin et al., 2011) proposed a new constitutive model of foam with only few parameters, where each parameter was a function of its density.

Often, in a design process, the engineers assume an elastic range of loading and, therefore, focus on investigating the elastic properties only (EN 14509), namely Young's moduli $E_{i}$, Kirchhoff's moduli $G_{i j}$, and Poisson's ratios $\nu_{i j}$, using conventional procedures based on tension, compression, shear and bending tests with standardized geometry. For example, a shear modulus $G$ of the polyurethane core in sandwich panels plays the crucial role in the functional response and load-bearing capacity, therefore its proper identification is a very important issue. The development of the suitable experimental method of the estimation of this parameter was undertaken by many authors (e.g. Chuda-Kowalska et al., 2010; Juntikka amd Hallstrom, 2007). However, foams can exhibit orthotropic properties depending on the direction of loading (Caliri et al., 2012). A very short elastic range and the fact that the same material may exhibit brittle, perfect plastic or hardened responses depending on the direction along which the load is applied can complicate determination of the mechanical response of foams. Nevertheless, more advanced analyses require appropriate constitutive modeling of the material, an analysis far beyond the elastic regime, a set of reliable parameters and following numerous experimental tests. Therefore, alternative techniques (e.g. thermal imaging, vibrations registration, X-ray computed microtomography, digital image correlation) are currently being exploited in order to complement the standard test procedure and obtain more accurate results (Roux et al., 2008; Zhang et al., 2012).

Among advanced techniques, digital image correlation (DIC) is a very useful and successful tool. It is often used as a measurement procedure within an inverse analysis for the characterization of material properties (Gajewski and Garbowski, 2014a,b; Garbowski et al., 2012; Maier et al., 2010). Herein, the inverse problem is applied to determine orthotropic elastic behavior of a closed-cell stiff polyurethane foam (i.e. core of a sandwich panel) with the density of $40 \mathrm{~kg} / \mathrm{m}^{3}$. This research serves as the preliminary step in the development of the full procedure for complete characterization of constitutive models of elasto-plastic foams. 


\section{Mixed experimental-numerical analysis}

\subsection{Preliminary remarks}

For sandwich panels, there exist several methods for the estimation of core shear properties. They are still under development. The most of them are based on a displacement measurement where the data are recorded using appropriate gauges. Standard indirect experimental methods of the determination of the Kirchhoff modulus $G$ of the core were presented in (EN 14509). They are based on bending and shear tests of panels in which the transverse displacement is measured on the assumption of the core material isotropy. In order to obtain more accurate results and the take into account the orthotropic effect, a direct experimental method called the double-lap shear test in combination with digital image correlation techniques is used in this paper. This combination results in very efficient and meaningful experimental data which later can be used as a counterpart to numerically computed responses by test simulation. In order to carry out the test simulation, a numerical model needs to be constructed and fed by parameters, which at this stage are unknown, so one may perform an iterative procedure for automatic update of the parameters.

Here, the experimental data are used simultaneously with the data numerically generated by the model. Such synergy exploits the best features of the experiment and its numerical simulation through sensitivity analysis. The computed sensitivities of measurable quantities with respect to model parameters explain if the test is properly designed and point out which parameters are activated through the test. The measurable quantity is the displacement field obtained from the side surface of the sample and the parameters are material constants embedded in the elastic orthotropic model of the foam.

Having both the experimental data and computed equivalents, one can minimize the discrepancy between these measurements by the least square technique. The iterative procedure leads to the set of parameters for which the numerical response is almost the same as the measured one. At this point, the procedure stops, and the calculated parameters are assumed to be the sought ones.

\subsection{Experimental setup}

As has already been mentioned in the previous section, sandwich panels with a polyurethane core and metal sheets are investigated. The three orthogonal material directions of the foam and the way of cutting off two samples from the manufactured panel for the double-lap shear test purpose are depicted in Fig. 1.

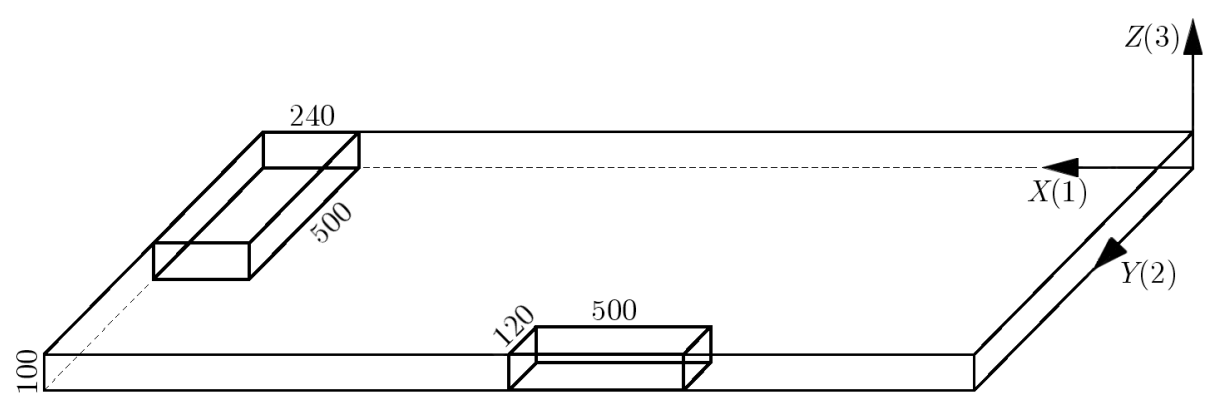

Fig. 1. Cut off specimens and the principal directions of the panel, $X(1), Y(2), Z(3)$

The double-lap shear test (Chuda-Kowalska et al., 2010) is used here as the experimental setup. The purpose of the examination is to obtain a uniform shear stress field within the sample and to minimize rotation and bending (as occurs in a single-block shear test) (Benderly et al., 2003). The laboratory setup for the double-lap shear test is illustrated in Fig. 2. 

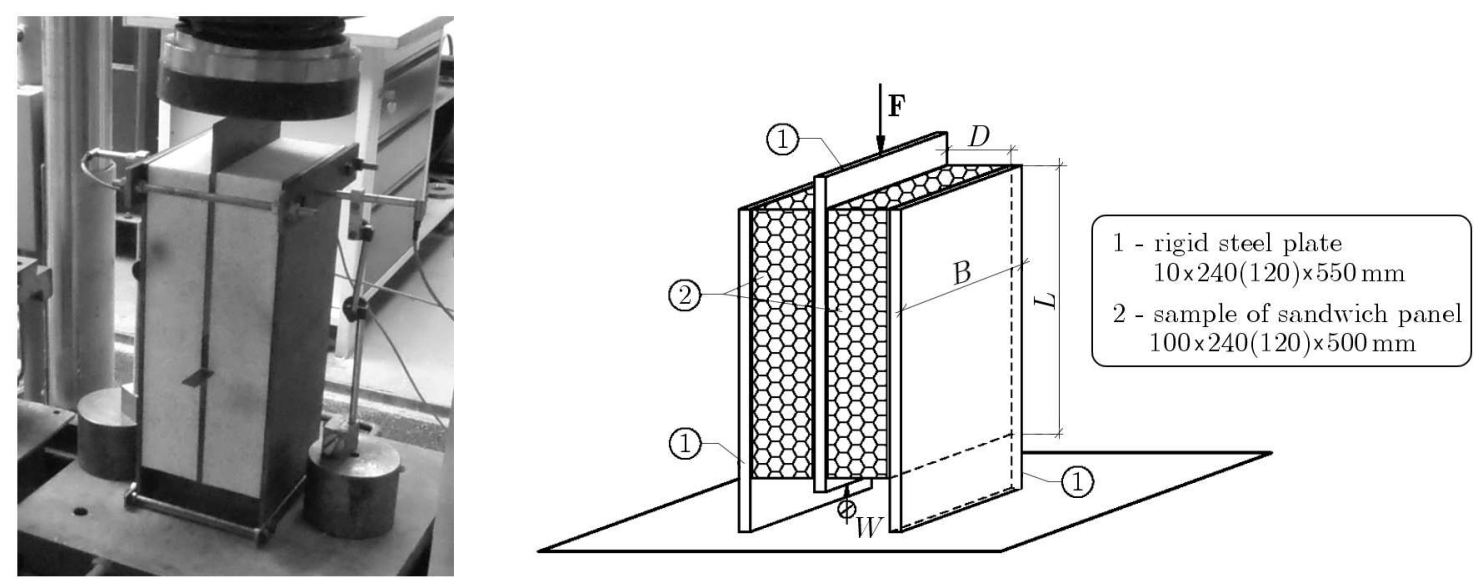

Fig. 2. The experimental setup of a double-lap shear test, photography and scheme of the sample with particular dimensions

The single sample is first properly cut off form the panel and later positioned between three rigid steel plates and glued to them. The force $F$ acts on the middle plate and the vertical displacement $W$ of the rigid plate is measured simultaneously. The quasi-static loading velocity is controlled by the strain rate, in this case $2 \mathrm{~mm} / \mathrm{min}$. The experiment is carried out on the Instron 8505 plus (http://www.instron.tm.fr) four-column frame servohydraulic fatigue testing machine with compressive force capacity of up to $1000 \mathrm{kN}$. This electronically controlled and versatile system can perform static, fatigue and dynamic tests on various materials.

\subsection{Digital image correlation}

In this research, the presented double-lap shear procedure is enhanced by digital image registration and correlation of the specimen surface in-plane deformations. This enrichment allows one, in the post-processing state by a digital image correlation algorithm, acquire a full-field displacement of the specimen surface. The digital recording starts exactly at the moment when the shear force is applied and continues until fracture of the specimen. Due to continuous recording the displacement field can be tracked in different time frames during the test, therefore the deformation field can be obtained for various force values.

The digital image correlation is an optical technique for the displacements or/and strains determination between two digitally recorded stages, comparing an original picture of the load free specimen with subsequent pictures of deformed configurations. This contactless measurement method is successfully used as an alternative to standard measurement devices, e.g. strain gauges. Here, however, the main benefit of DIC application is due to the amount of measurement to be done on the specimen surface, which helps one to regularize an inverse procedure. The DIC algorithm calculates, on the basis of the undeformed picture, the deformed picture and then checks the difference between the computed data and the image recorded during the test. The discrepancy is iteratively reduced by minimization of the main objective, i.e. correlation criterion, see Eqs. (2.1). In the acquired digital images, usually in a gray scale, color intensities are stored as numbers in each pixel, where each value ranges from 0 to 255 . The quantities between 0 (black) and 255 (white) represent different shades of gray.

The discrepancies between the two sets of data in the deformed state (computed and recorded) are determined by a correlation function. According to the review paper of Pan et al. (2009), the correlation function, sometimes called the correlation criterion, can be categorized into the two main families, i.e. cross-correlation (CC) and sum-squared differences (SSD) criterion. Those functions, depending on its compliance, can exclude the error due to a change in the 
lighting condition during image recording. For instance, the zero-normalized CC (ZNCC) and zero-normalized SDD (ZNSSD) criteria $C_{i}$, see Pan et al. (2009), are given by the formulas

$$
\begin{aligned}
C_{\mathrm{ZNCC}} & =\sum_{i=-M}^{M} \sum_{j=-M}^{M} \frac{\left[f\left(x_{i}, y_{j}\right)-f_{m}\right]\left[g\left(x_{i}^{\prime}, y_{j}^{\prime}\right)-g_{m}\right]}{\Delta f \Delta g} \\
C_{\mathrm{ZNSSD}} & =\sum_{i=-M}^{M} \sum_{j=-M}^{M}\left(\frac{f\left(x_{i}, y_{j}\right)-f_{m}}{\Delta f}-\frac{g\left(x_{i}^{\prime}, y_{j}^{\prime}\right)-g_{m}}{\Delta g}\right)^{2}
\end{aligned}
$$

where $f\left(x_{i}, y_{i}\right)$ is the gray pixel value at the position $(x, y)$ of the load free image and $g\left(x^{\prime}, y^{\prime}\right)$ is the gray pixel value at the position $\left(x^{\prime}, y^{\prime}\right)$ of the deformed state image. $M$ is a half of the pixel subset dimension (horizontal and vertical direction dimensions are usually the same). In formulas (2.1), the mean pixel values in the reference image $f_{m}$ and in the deformed one $g_{m}$ are calculated using the corresponding expressions

$$
f_{m}=\frac{1}{(2 M+1)^{2}} \sum_{i=-M}^{M} \sum_{j=-M}^{M} f\left(x_{i}, y_{i}\right) \quad g_{m}=\frac{1}{(2 M+1)^{2}} \sum_{i=-M}^{M} \sum_{j=-M}^{M} g\left(x_{i}^{\prime}, y_{i}^{\prime}\right)
$$

The norms $\Delta f$ and $\Delta g$ of the load free and the deformed image are calculated by the formulas

$$
\Delta f=\sqrt{\sum_{i=-M}^{M} \sum_{j=-M}^{M}\left[f\left(x_{i}, y_{i}\right)-f_{m}\right]^{2}} \quad \Delta g=\sqrt{\sum_{i=-M}^{M} \sum_{j=-M}^{M}\left[g\left(x_{i}^{\prime}, y_{i}^{\prime}\right)-g_{m}\right]^{2}}
$$

To eliminate the lack of uniqueness of the DIC solution, a subset of the pixels (usually with dozens pixels in height and width) in both images are picked up to be compared in a single step of the correlation algorithm. This small subset of the pixels is called the zone of interest (ZOI), and its center represents one node of the tracked displacement grid. The group of ZOIs builds the region of interest (ROI) which defines the sought displacement field. Both quantities, in the $x$ and $y$ orientations, are performed. The example of ZOI grid within the picked ROI is illustrated in Fig. 3.

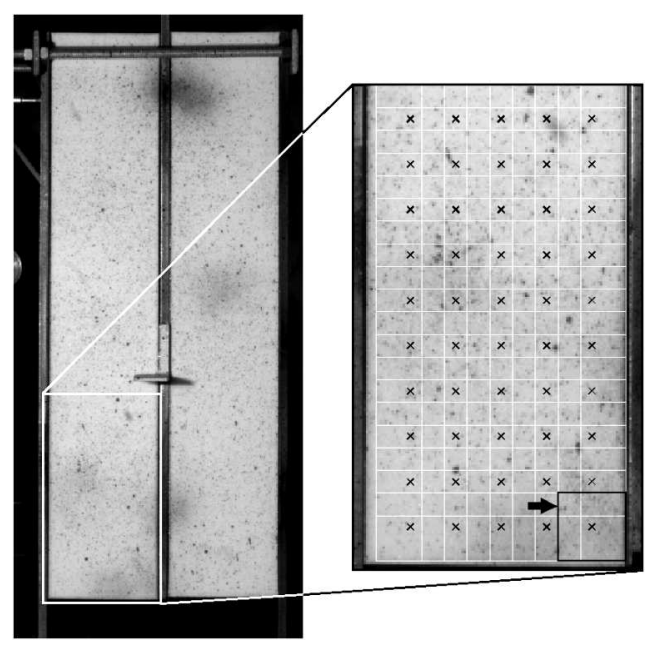

Fig. 3. An example of the recorded image in load free configuration with the separated region of interest, divided into the ZOI grid (overlapping white squares with centers in black crosses). Single ZOI is denoted by the black arrow within a black square 

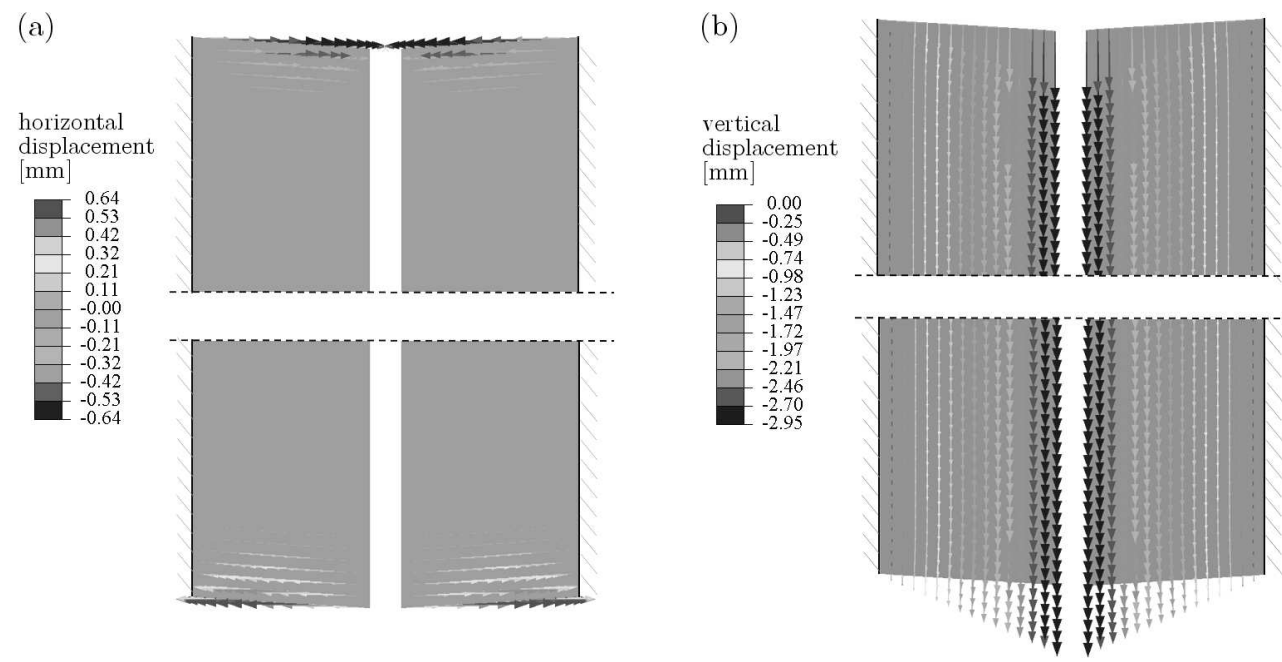

Fig. 4. The vertical (a) and horizontal (b) displacement fields for the pseudo-experimental setup of parameters

\subsection{Finite element model and the adopted constitutive model}

The experimental test is here simulated by the finite element (FE) model, which serves as a numerical counterpart in the material characterization procedure. The DIC measurable quantities and corresponding computed values (i.e. the in-plane surface displacements) are used to build the discrepancy between the two systems: experimental and numerical. Geometry, boundary conditions and loads in the simulation are adapted from the real test. Due to symmetry, simulations are performed on a half of the real experiment structure with appropriate symmetry boundary conditions. Two experimental setups are modeled here, within a separate FE force control protocol analysis. This is due to the fact that the specimens cut off in two main orthotropy directions differ not only because of material orientation but also because of their dimensions. The specimens cut off across and along panel length as shown in Fig. 1. They have dimensions of $100 \times 240 \times 550$ and $100 \times 120 \times 550 \mathrm{~mm}$, respectively. This implies the use of two separate and distinct $\mathrm{FE}$ models on each step of the iterative material characterization procedure. The solid material domain is meshed by 25054 -node bilinear plane stress elements.

A linear elasticity in an orthotropic material is defined by giving the engineering constants: three tensile moduli: $E_{1}, E_{2}, E_{3}$, shear moduli: $G_{12}, G_{13}, G_{23}$ and Poisson's ratios: $\nu_{12}, \nu_{13}, \nu_{23}$. These parameters define the elastic compliance according to

$$
\left[\begin{array}{c}
\varepsilon_{11} \\
\varepsilon_{22} \\
\varepsilon_{33} \\
\gamma_{12} \\
\gamma_{13} \\
\gamma_{23}
\end{array}\right]=\left[\begin{array}{cccccc}
1 / E_{1} & -\nu_{21} / E_{2} & -\nu_{31} / E_{3} & 0 & 0 & 0 \\
-\nu_{12} / E_{1} & 1 / E_{2} & -\nu_{32} / E_{3} & 0 & 0 & 0 \\
-\nu_{13} / E_{1} & -\nu_{23} / E_{2} & 1 / E_{3} & 0 & 0 & 0 \\
0 & 0 & 0 & 1 / G_{12} & 0 & 0 \\
0 & 0 & 0 & 0 & 1 / G_{13} & 0 \\
0 & 0 & 0 & 0 & 0 & 1 / G_{23}
\end{array}\right]\left[\begin{array}{c}
\sigma_{11} \\
\sigma_{22} \\
\sigma_{33} \\
\sigma_{12} \\
\sigma_{13} \\
\sigma_{23}
\end{array}\right]
$$

In general, the relationship between Poisson's ratios $\nu_{i j}$ and $\nu_{j i}$ is defined by

$$
\frac{\nu_{i j}}{E_{i}}=\frac{\nu_{j i}}{E_{j}}
$$

Since the measurements are performed on the external surface of the sample, the plain stress condition is assumed here and, consistently, modeled in the small strain regime. 
For plane stress conditions in 1-3 plane (sample $100 \times 120 \times 500 \mathrm{~mm}-$ Fig. 1 ), the material stiffness matrix reads

$$
\mathbf{D}=\frac{1}{1-\nu_{12} \nu_{21}}\left[\begin{array}{ccc}
E_{1} & E_{3} & 0 \\
E_{1} & E_{3} & 0 \\
0 & 0 & G_{13}\left(1-\nu_{13} \nu_{31}\right)
\end{array}\right]
$$

Here only the four elastic constant among nine are active, namely: $E_{1}, E_{3}, \nu_{13}$ and $G_{13}$. The remaining three parameters, namely: $E_{2}, \nu_{23}$ and $G_{23}$, are active in 2-3 plane (sample $100 \times 240 \times 500 \mathrm{~mm}-$ Fig. 1 ). The last two parameters, $G_{12}$ and $\nu_{12}$, can be computed analytically by the simple formula proposed by Campbell (1961)

$$
\frac{1}{G_{12}}=\frac{1+\nu_{12}}{E_{1}}+\frac{1+\nu_{21}}{E_{2}}
$$

The only missing parameter, namely Poisson's ratio $\nu_{12}$, is assumed (based on the observations and laboratory testing) to play a marginal role herein, therefore its value is set to 0.01 .

\subsection{Inverse analysis}

Once the experimental setup is selected in the way that the measurable quantities are sensitive to model parameters, a mathematical program is employed for iterative computation of the sought parameters. Here, the trust region (TR) algorithm is utilized for minimization of the objective function within the least square frame. In the family of trust region algorithms, there is no need to compute the step length because the solution is searched on a local quadratic approximation of the objective function bounded by a domain called the trust region. The implemented preconditioning method and gradient scaling techniques allow one to use box constrains on the parameter space.

The TR algorithm in general searches for the solution in the space spanned between the Gauss-Newton direction and the steepest descent direction. Such an approach allows one to find the solution in just few iterations (provided that the starting point is relatively close to the optimal solution) and does not require (as already mentioned) the expensive step length computations.

\section{Results and discussion}

\subsection{Sensitivity analysis}

The presented research consists of several integral parts of material identification, i.e. (1) double-lap shear test of the foam sample enhanced by the DIC measurements, (2) image correlation software, (3) inverse procedure frame with the minimization algorithm, (4) numerical model of experiment and finally, (5) selected constitutive law (suitable in a certain range of the foam behavior) with parameters to be characterized.

The determination of the unknown material variables constitutes the material mechanical behavior. The parameters selected in this investigation are introduced in the first column of Table 1 with its reference values for the pseudo-experimental purpose (more detailed explanation is presented in Section 3.2).

The influence of particular material variables on the system can be simply evaluated by a sensitivity analysis. Here, the sensitivities of measurable quantities with respect to the foam parameters are computed and presented in Figs. 5a-d, the sensitivity values are calculated according to the formula

$$
s_{i}^{j}=\left|\frac{1}{\delta}\left(1-\frac{\left\|\mathbf{u}_{i}^{j}\right\|}{\left\|\mathbf{u}_{\mathrm{REF}}^{j}\right\|}\right)\right|
$$


Table 1. The reference values of parameters used for sensitivity and inverse calculations for Case I and Case II

\begin{tabular}{|l|c|c|}
\hline Parameter & Case I & Case II \\
\hline \hline$E_{1}[\mathrm{MPa}]$ & 14.9 & 14.9 \\
\hline$E_{2}[\mathrm{MPa}]$ & 6.59 & 6.59 \\
\hline$E_{3}[\mathrm{MPa}]$ & 3.22 & 3.22 \\
\hline$G_{31}[\mathrm{MPa}]$ & 2.20 & 2.20 \\
\hline$G_{32}[\mathrm{MPa}]$ & 2.50 & 2.50 \\
\hline$\nu_{31}[-]$ & 0.01 & $\underline{0.25}$ \\
\hline$\nu_{32}[-]$ & 0.01 & $\underline{0.40}$ \\
\hline
\end{tabular}

and

$$
\mathbf{u}_{i}^{j}=\sum_{k=1}^{n} \mathbf{u}_{i}^{k j} \quad i=1,2,3, \ldots, 7 \quad j=x, y \quad k=1,2, \ldots, n
$$

where $i$ is the number of parameter, $j$ is the direction of the considered displacement, $k$ is the number of nodes in measurable displacements, $u_{i}^{k j}$ is the displacement field, $\delta=0.01$ is a small perturbation of the parameter $x_{i}, u_{\mathrm{REF}}^{j}$ is the displacement calculated using the finite element model fed with the reference material parameters shown in Table 1 . The reference values $E_{1}, E_{2}$, $E_{3}, G_{31}, G_{32}$ in both Case I and Case II are taken from the previous experimental trials. Poisson's ratios $\nu_{31}$ and $\nu_{31}$, according to preliminary tests, are expected to be close to 0 , therefore in Case I they equal 0.01. In Case II, nonzero values are assumed, 0.25 and 0.40 respectively, to check how this change will modify the sensitivities outcome. The bars in Fig. 5 illustrate how the change of each parameter by a small perturbation $\delta$ affects the effective value of measurable quantities.

Case I. Figure 5a indicates higher sensitivities of horizontal displacements with respect to $E_{1}, E_{2}$ and smaller for $G_{31}, G_{32}$, the other sensitivities are negligible. In the vertical direction, see Fig. 5b, the sensitivities of the measurable quantities with respect to $G_{31}$ and $G_{32}$ have the biggest values (as expected), the contribution of the other parameters to the vertical displacement measurements are again negligible.

Case II. The results presented in Fig. 5d correspond to those in Fig. 5b with no qualitative differences, however between Fig. 5c and Fig. 5a, some meaningful changes can be observed. Including nonzero Poisson's ratios in Case II results in a noticeable increase in the sensitivity with respect to $E_{1}, E_{2}$ and a significant change with respect to $E_{3}, \nu_{31}$ and $\nu_{32}$, but only for the horizontal displacements measurements.

\subsection{Inverse analysis}

Once the sensitivity analysis has been performed, the further step can be made, namely the validation through a pseudo-experimental approach where the data taken as the experimental ones are computed numerically. The measurable quantities are calculated based on the known parameters which are later sought in the inverse problem framework. This technique allows one to check if the numerical system is able to converge to the sought (known a priori) solution.

This pseudo-experimental verification is carried out on the numerical model different from the one used for the identification in terms of meshes. Figures 6 and 7 illustrate two successful characterizations of the foam for the same selected set of parameters, however with different initial points of the inverse analysis. The graphs compose convergence plots of normalized parameters (a) and corresponding cost function values (b). The horizontal axis in both cases expresses the number of the inverse analysis step (iterations). 

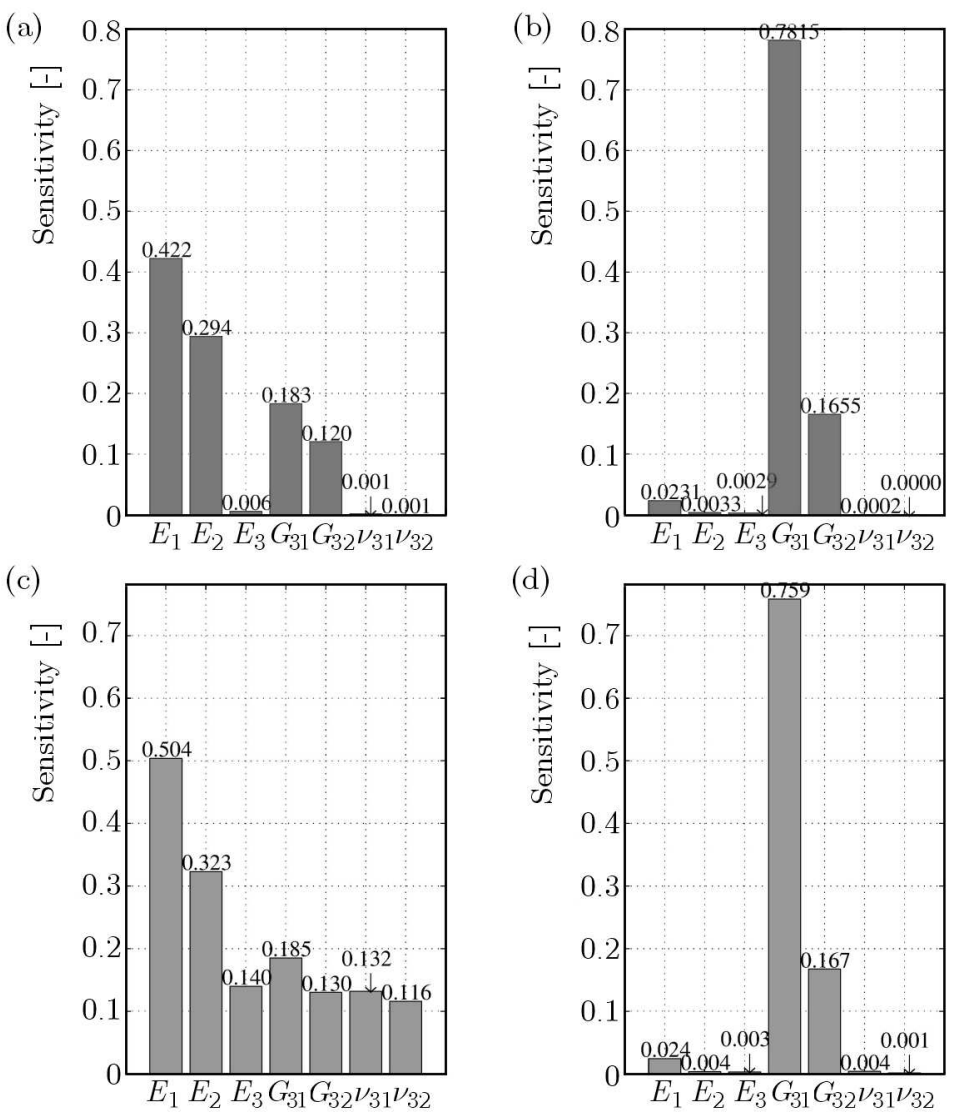

Fig. 5. Sensitivity plots of FE computation of the displacements field with respect to constitutive material parameters $\left(E_{1}, E_{2}, E_{3}, G_{31}, G_{32}, \nu_{31}, \nu_{32}\right)$. Case I, sensitivities of the horizontal (a) and the vertical (b) displacements. Case II, sensitivities for the horizontal (c) and the vertical (d) displacements
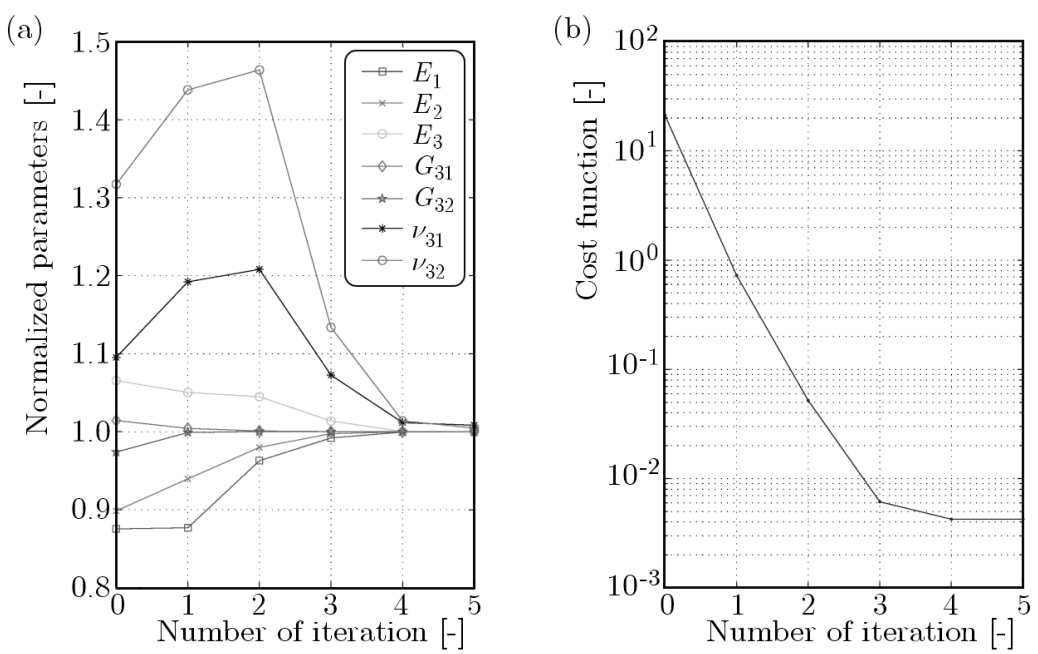

Fig. 6. Case I. Characterization of the foam with a noisy pseudo-experimental data $\pm 1 \mu \mathrm{m}$; (a) convergence of the normalized parameters and (b) corresponding cost function values

As is shown in Figs. 6, 7, after several iterations, the randomly initialized values of the material variables are properly converging to the normalized values $1.0\left(x_{i} / x_{i}^{r e f}\right)$. This means that the parameters which are computed by the inverse analysis $x_{i}$ correspond to the a priori assumed reference ones $x_{i}^{\text {ref }}$ (in pseudo-experiment). The discrepancy between the two displacement fields is iteratively reduced by the optimization algorithm, therefore the cost function 

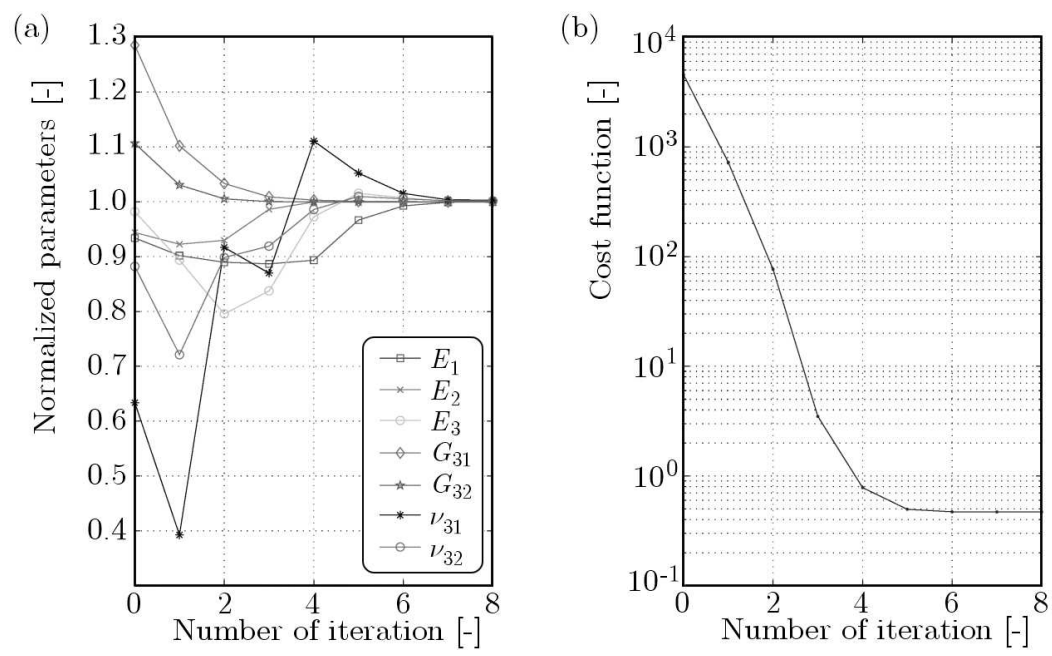

Fig. 7. Case II. Characterization of the foam with a noisy pseudo-experimental data $\pm 10 \mu \mathrm{m}$;

(a) convergence of the normalized parameters and (b) corresponding cost function values

progressively decreases through the steps. It can be seen in Fig. $6 \mathrm{~b}, 7 \mathrm{~b}$ that firstly the curves sharply fall to transit into plateau, which is a characteristic shape when the noised measurable data are considered. Here, the $1 \mu \mathrm{m}$ and $10 \mu \mathrm{m}$ uniform distribution of random noise is added to the measurements, respectively for Case I (Fig. 6) and Case II (Fig. 7) in order to mimic the real noisy measurements.

\section{Conclusions}

The identification of material parameters is a complex engineering problem especially when the parameters can not be found directly or many tests have to be employed for characterization of a single material. To obtain a proper backward solution, a number of tools should be framed into the robust characterization procedure. Such a system requires (1) sensitive measurements from the experiment, (2) numerical simulation model, which mimics performance of the real system and (3) minimization algorithm. To avoid ill-posed problems, e.g. lack of uniqueness of the sought solution, a sufficient number of measurable data should be considered. Also a pseudo-experimental approach is good preliminary verification. In the discussed numerical-pseudo-experimental investigation, the sensitivities of the measurements with respect to all active model parameters have been registered and introduced in the paper.

On the basis of the investigation, several remarks can be noted. Firstly, for the sensitivities, if Poisson's ratios are close to 0 (Case I), the system shows minimal (negligible) sensitivity to those variables, however if the ratios are set (in the pseudo-experimental approach) to nonzero values (Case II), the sensitivity increases significantly. Due to that, even when a bigger value of noise is introduced $(10 \mu \mathrm{m}), \nu_{31}$ and $\nu_{32}$ are easily reconstructed to their reference values. Therefore, it can be claimed that the characterization of seven parameters, namely $E_{1}, E_{2}, E_{3}, G_{31}, G_{32}$, $\nu_{31}, \nu_{32}$, would be successfully accomplished by making use of the mixed numerical-experimental method.

Finally, it has to be underlined that only the two tests have been conducted in the perpendicular material axis, thus there is no possibility to determine the missing unknowns linked with the third plane, i.e. $G_{12}, \nu_{12}$, which are inactive in the performed protocols. Due to manufactured dimensions of the panels, the third orthogonal direction cannot be considered in a similar experimental setup, though the values can be successfully calculated from simplified formulas (2.5). 
In overall, to describe the orthotropic constitutive behavior of the material in $3 \mathrm{D}$, the twelve parameters as stated in Eq. (2.2) have to be assigned. This number reduces to nine according to Eq. (2.3). Here, the proposed procedure successfully characterizes the seven unknown elastic parameters of the foam material, therefore further investigation has to be conducted. Within the proposed framework, the most straightforward would be to conduct the third, additional test with a smaller specimen (due to small dimension of the panel in the out-of-plane direction), however such an approach extends the number of tests performed to characterize the described material, which is in conflict with the general aim of the presented paper.

\section{References}

1. Avalle M., Belingardi G., IbBa A., 2007, Mechanical models of cellular solids: parameters identification from experimental tests, International Journal of Impact Engineering, 34, 3-27

2. Benderly D., Zafran J., Putter S., 2003, Shear testing of polymer foams, Journal of Testing and Evaluation, 31, 5, 405412

3. BŁaszczuk J., Pozorski, Z., 2012, The analysis of the influence of core compression effect on the determination of the shear modulus of the sandwich panel core, Scientific Research of the Institute of Mathematics and Computer Science, 2, 11, 5-13

4. Caliri Jr M.F., Soares G.P., Angélico R.A., Canto R.B., Tita V., 2012, Study of an anisotropic polymeric cellular material under compression loading, Materials Research, 15, 3, $359-364$

5. Campbell J.G., 1961, The in-plane elastic constants of paper, Australian Journal of Applied Science, 12, 3, 356-357

6. Chuda-Kowalska M., Pozorski Z., Garstecki A., 2010, Experimental determination of shear rigidity of sandwich panels with soft core, Proceedings of the 10th International Conference Modern Buildings Materials, Structures and Techniques, Vilnius Gediminas Technical University Press, 56-63

7. EN 14509 Self-supporting double skin metal faced insulating panels - Factory made products Specifications

8. Gajewski T., Garbowski T., 2014a, Calibration of concrete parameters based on digital image correlation and inverse analysis, Archives of Civil and Mechanical Engineering, 14, 1, 170-180

9. Gajewski T., Garbowski T., 2014b, Mixed experimental/numerical methods applied for concrete parameters estimation, Recent Advances in Computational Mechanics - Proceedings of the 20th International Conference on Computer Methods in Mechanics, CMM 2013, Łodygowski T., Rakowski J. and Litewka P. (Edit.), CRC Press, 293-302

10. Garbowski T., Maier G., Novati G., 2012, On calibration of orthotropic elastic-plastic constitutive models for paper foils by biaxial tests and inverse analyses, Structural and Multidisciplinary Optimization, 46, 1, 111-128

11. Gibson L.J., Ashby M.F., 1997, Cellular Solids. Structure and Properties, Cambridge University Press, 2nd ed., ISBN 0521499119

12. Janus-Michalska M., 2009, Micromechanical model of auxetic cellural materials, Journal of Theoretical and Applied Mechanics, 47, 4, 737-750

13. Janus-Michalska M., Pęcherski R.B., 2003, Macroscopic properties of open-cell foams based on micromechanical modelling, Technische Mechanik, 23, 2/4, 221-231

14. Juntikka R., Hallstrom S., 2007, Shear characterization of sandwich core materials using fourpoint bending, Journal of Sandwich Structures and Materials, 9, 67-94 
15. Kordzikowski P., Janus-Michalska M., Pęcherski R.B., 2005, Specification of energy-based criterion of elastic limit states for cellular materials, Archives of Metallurgy and Materials, 50, 619-634

16. Liu Q., Subhash G., 2004, A phenomenological constitutive model for foams under large deformations, Polymer Engineering and Science, 44, 3, 463-473

17. Maier G., Bolzon G., Buljak V., Garbowski T., Miller B., 2010, Chapter 24. Synergistic combinations of computational methods and experiments for structural diagnosis, [In:] Computer Methods in Mechanics. Lectures of the CMM 2009, M. Kuczma, K. Wilmanski (Edit.), SpringerVarlag Berlin Heidelberg, 453-476

18. Maier G., Buljak V., Garbowski T., Cocchetti G., Novati G., 2014, Mechanical characterization of materials and diagnosis of structures by inverse analyses: some innovative procedures and applications, International Journal of Comutational Methods, 11, 3, 1343002, DOI: 10.1142/S0219876213430020

19. Mills N., 2007, Polymer foams handbook. Engineering and Biomechanics Applications and Design Guide, Butterworth-Heinemann, ISBN-10: 0750680695, ISBN-13: 978-0750680691

20. Murin J., Kompiš V., Kutiš V., 2011, Computational Modelling and Advanced Simulations, Springer

21. Ozturk U.E., Anlas G., 2009, Energy absorption calculations in multiple compressive loading of polymeric foams, Materials and Design, 30, 15-22

22. PAn B., Qian K., Xie H., Asundi A., 2009, Two-dimensional digital image correlation for inplane displacement and strain measurement: a review, Measurement Science and Technology, 20, 6

23. Roux S., Hild F., Viot P., Bernard D., 2008, Three-dimensional image correlation from X-ray computed tomography of solid foam, Composites: Part A: Applied Science and Manufacturing, 39, 1253-1265

24. Studziński R., Pozorski Z., Garstecki A., 2013, Sensitivity analysis of sandwich beams and plates accounting for variable support conditions, Bulletin of the Polish Academy of Sciences Technical Sciences, 61, 201-210

25. Subramanian N., Sankar B.V., 2012, Evaluation of micromechanical methods to determine stiffness and strength properties of foams, Journal of Sandwich Structures and Materials

26. Valdevit, L., And Hutchinson, J.W., And Evans, A.G., 2004, Structurally optimized sandwich panels with prismatic cores, International Journal of Solids and Structures, 41, 51055124

27. Zhang S., Dulieu-Barton J.M., Fruehmann R.K., Thomsen O.T., 2012, A methodology for obtaining material properties of polymeric foam at elevated temperatures, Experimental Mechanics, 52, 3-15 